\title{
Interpretation of Continuous-time Autoregressive Processes as Random Exponential Splines
}

\author{
Julien Fageot, John Paul Ward, and Michael Unser \\ Biomedical Imaging Group \\ Ecole polytechnique fédérale de Lausanne \\ CH-1015 Lausanne VD, Switzerland \\ Email: julien.fageot@epfl.ch
}

\begin{abstract}
We consider the class of continuous-time autoregressive (CAR) processes driven by (possibly non-Gaussian) Lévy white noises. When the excitation is an impulsive noise, also known as compound Poisson noise, the associated CAR process is a random non-uniform exponential spline. Therefore, Poissontype processes are relatively easy to understand in the sense that they have a finite rate of innovation. We show in this paper that any CAR process is the limit in distribution of a sequence of CAR processes driven by impulsive noises. Hence, we provide a new interpretation of general CAR processes as limits of random exponential splines. We illustrate our result with simulations.
\end{abstract}

\section{INTRODUCTION}

Continuous autoregressive-called continuous AR, or CAR-models are widely used in various domains, such as quantitative finance, astronomy, and signal processing, to describe time-varying processes. Among the family of CAR models, the Gaussian ones are by far the most popular and the easiest to specify [1]. The family can also be extended to allow for non-Gaussian sparse models [2], [3], [4].

The continuous-domain processes studied in this paper are solutions of a stochastic differential equation driven by a Lévy noise. Prominently, they include the family of CAR processes, which are stationary. Moreover, our theory is general enough to include Lévy processes, which are not stationary but have stationary increments. Therefore, with some abuse of terminology, we include Lévy-type processes when we mention the class of CAR processes.

Among the family of studied processes, very different behaviors are exhibited. For instance, the sample paths of Brownian motion are fractals [5]. On the other hand, compound Poisson processes, which are also part of the Lévy family, are piecewise constant [6]. Hence, they have a finite rate of innovation [7].

Splines are continuous-domain functions defined from a discrete set of data. In the context of sampling theory, they provide a link between continuous- and discrete-domain signals. A spline is usually defined by a sequence of knots and sample values, and a set of basis functions.

Due to their link with ordinary differential operators, we focus on the family of exponential splines [8] and investigate their connections with CAR processes. We can already remark that a compound Poisson Lévy process, having piecewise constant sample paths, has a spline interpretation. The knots

978-1-4673-7353-1/15/\$31.00 @ 2015 IEEE of a sample path are the locations of the impulses of the underlying noise and the basis function is the unit step function [6]. Moreover, a stochastic process driven by an impulsive, or compound Poisson, noise can similarly be described using splines [9].

In general, non-Poisson CAR processes do not have such a direct spline-based interpretation, yet we shall demonstrate that a CAR process is the limit in distribution of a sequence of impulse-driven CAR processes. In other words, a CAR process is the limit in distribution of random exponential splines. This gives a new interpretation of CAR processes in terms of spline theory. Interestingly, the principle of modeling a noise as "a large number of closely spaced and very short impulses" was used in the early days of signal processing by Bode and Shannon [10, Section VI].

In Section II, we introduce the class of Lévy driven CAR processes. In Section III, we recall basic facts about exponential splines. This allows us to connect exponential splines with CAR processes driven by compound Poisson noises. Our main contribution is presented in Section IV: we show that any CAR process is the limit of a sequence of impuse-driven CAR processes. Simulations of this result are given in Section V. We conclude in Section VI.

\section{Continuous AR Processes}

\section{A. Generalized Stochastic Processes in $\mathcal{S}^{\prime}(\mathbb{R})$}

We introduce the general class of continuous-domain, Lévydriven AR processes within the framework of generalized random processes, which is based on the seminal work of Gelfand and Vilenkin [11].

Generalized random processes include classical stochastic processes, such as Lévy processes. It is also possible to properly define white noises and their derivatives, which are not available in more traditional approaches for stochastic processes.

A generalized random process is a random element of the space $\mathcal{S}^{\prime}(\mathbb{R})$ of tempered generalized functions of Schwartz. In general, such a process $s$ cannot be evaluated at a given time $t$. It can only be observed through window functions $\varphi$ in $\mathcal{S}(\mathbb{R})$, the space of rapidly decaying smooth functions. The duality product $\langle s, \varphi\rangle$ is then a real random variable. The characteristic functional of a generalized random process $s$ is the Fourier 
transform of its probability law, defined for $\varphi \in \mathcal{S}(\mathbb{R})$ by

$$
\widehat{\mathscr{P}}_{s}(\varphi):=\mathbb{E}\left[\mathrm{e}^{\mathrm{j}\langle s, \varphi\rangle}\right] .
$$

The characteristic functional of $s$ contains all of the statistical characteristics of $s$. For instance, a process $s$ is stationary if and only if $\widehat{\mathscr{P}}_{s}(\varphi(\cdot-\tau))=\widehat{\mathscr{P}}_{s}(\varphi)$ for every $\varphi \in \mathcal{S}(\mathbb{R})$ and $\tau \in \mathbb{R}$. Moreover, the characteristic function $\Phi_{X}$ of the random variable $X=\langle s, \varphi\rangle$ can be deduced from the characteristic functional of $s$ by the relation $\Phi_{X}(\omega):=\mathbb{E}\left[\mathrm{e}^{\mathrm{j} \omega X}\right]=$ $\widehat{\mathscr{P}}_{s}(\omega \varphi)$. (Similar considerations can be made for every finitedimensional marginal of s.) Note moreover that, for a linear operator L from $\mathcal{S}^{\prime}(\mathbb{R})$ to $\mathcal{S}^{\prime}(\mathbb{R})$ with adjoint $\mathrm{L}^{*}$ from $\mathcal{S}(\mathbb{R})$ to itself, one has by duality

$$
\widehat{\mathscr{P}}_{\mathrm{L} s}(\varphi)=\mathbb{E}\left[\mathrm{e}^{\mathrm{j}\langle\mathrm{L} s, \varphi\rangle}\right]=\mathbb{E}\left[\mathrm{e}^{\mathrm{j}\left\langle s, \mathrm{~L}^{*} \varphi\right\rangle}\right]=\widehat{\mathscr{P}}_{s}\left(\mathrm{~L}^{*} \varphi\right)
$$

for every $\varphi \in \mathcal{S}(\mathbb{R})$.

A characteristic functional fully characterizes the probability law of a process. Moreover, the Minlos-Bochner theorem ensures that a functional on $\mathcal{S}(\mathbb{R})$ is the characteristic functional of a generalized random process if and only if it is continuous, positive-definite and takes value 1 at $\varphi=0[9$, Theorem 3.9]. The fact that we can apply this result is due to the nuclear structure of $\mathcal{S}(\mathbb{R})$ [9, Section 3.1].

\section{B. Lévy White Noise}

A Lévy white noise is often described as the (weak) derivative of a Lévy process. Moreover, a Lévy process is completely characterized by the law of its value at time $t=1$, which is infinitely divisible. Consequently, there is a one to one correspondence between Lévy white noises and infinitely divisible random variables. A random variable is infinitely divisible if, for all $n$, it can be written as a sum of $n$ independent and identically distributed (i.i.d.) random variables [12]. If $X$ is infinitely divisible, its characteristic function can be written $\Phi_{X}(\omega)=\mathrm{e}^{f(\omega)}$ where $f(\omega)$ is called the Lévy exponent of $X$. In this paper, we only consider random variables $X$ such that $\mathbb{E}\left[|X|^{\epsilon}\right]<\infty$ for some $\epsilon>0$. Note that $\epsilon$ can be arbitrarily small, hence this condition is satisfied for any probability law used in practice. If a Lévy exponent $f$ of an infinitely divisible random variable has a finite-moment of order $\epsilon>0$, then it is called a Lévy-Schwartz exponent [13, Section 3].

It is interesting to recall that any Lévy exponent $f(\omega)$ can be decomposed into a Gaussian part and a pure jump part thanks to the Lévy-Khintchine theorem [12, Theorem 8.1]. In this paper, we shall only make a use of the following result

Proposition 1 (Lemma 7.9, [12]). Let $X$ be infinitely divisible, with Lévy exponent $f(\omega)$. Then, the function $\mathrm{e}^{\tau f(\omega)}$ is a valid characteristic function for every $\tau>0$.

A discrete-domain white noise is an i.i.d. vector. Let $\mathbf{X}=\left(X_{1}, \ldots, X_{N}\right)$ be an i.i.d. infinitely divisible vector, with Lévy exponent (the common one of the $X_{i}$ ) $f(\omega)$. Then, the characteristic function of $\mathbf{X}$ is

$$
\Phi_{\mathbf{X}}(\boldsymbol{\omega})=\exp \left(\sum_{n=1}^{N} f\left(\omega_{n}\right)\right)
$$

for all $\boldsymbol{\omega}=\left(w_{1}, \ldots, w_{N}\right) \in \mathbb{R}^{N}$. By analogy, we define a general continuous-domain white noise:

Definition 2. Let $f(\omega)$ be a Lévy-Schwartz exponent. A Lévy white noise $w$ with Lévy exponent $f(\omega)$ is a generalized random process in $\mathcal{S}^{\prime}(\mathbb{R})$ with characteristic functional of the form

$$
\widehat{\mathscr{P}}_{w}(\varphi)=\exp \left(\int_{\mathbb{R}} f(\varphi(t)) \mathrm{d} t\right)
$$

for all $\varphi \in \mathcal{S}(\mathbb{R})$.

Note the strong connection between (3) and (4), where the sum over $n$ is replaced by an integral over $t$. When $f(\omega)$ is a Lévy-Schwartz exponent, it is known that the characteristic functional in (4) defines a generalized random process in $\mathcal{S}^{\prime}(\mathbb{R})[13$, Section 3].

A Lévy white noise is stationary i.e., $w(\cdot-\tau)$ has the same law as $w$ for every $\tau$, and independent at every point i.e., $\left\langle w, \varphi_{1}\right\rangle$ and $\left\langle w, \varphi_{2}\right\rangle$ are independent if $\varphi_{1}$ and $\varphi_{2}$ have disjoint supports.

\section{Continuous AR Processes}

A Lévy-driven CAR process is a solution of the stochastic differential equation

$$
P(\mathrm{D}) s=w
$$

where $P$ is a polynomial, $\mathrm{D}$ is the derivative operator, and $w$ is a Lévy noise. Stricly speaking, CAR processes are the stationary solutions of (5). As we shall see, this corresponds to the case where $P(X)$ has no purely imaginary roots. However, as we explained in the introduction, we extend the class of studied processes to possibly non-stationary processes, under the condition that they have stationary increments. Lévy processes, solution of $\mathrm{D} s=w$, are the prototypical example of such processes.

Formally, we have the relation $s=P(\mathrm{D})^{-1} w$. However, one has to be careful in the inversion of the operator $P(D)$. One solution is to find a left-inverse of the adjoint operator $P(\mathrm{D})^{*}$ such that the functional $\varphi \mapsto \widehat{\mathscr{P}}_{w}\left(P(D)^{*-1} \varphi\right)$ is a welldefined characteristic functional. Then, we can use the MinlosBochner theorem to define the associated process $s$. This approach was used in [9]. We recall here a brief exposition of the construction of CAR processes in the framework of generalized random processes. More details can be found in [9], [14].

Let $P(X):=\left(\prod_{i=1}^{N_{1}}\left(\alpha_{i}-X\right)\right)\left(\prod_{k=1}^{N_{2}}\left(\mathrm{j} \omega_{k}-X\right)\right)$ where $\alpha_{i} \in \mathbb{C}$ satisfies $\operatorname{Re}\left(\alpha_{i}\right) \neq 0$ and $\omega_{k} \in \mathbb{R}$. Then, since $\mathrm{D}^{*}=$ $-\mathrm{D}$, we have

$$
P(\mathrm{D})^{*}=\left(\prod_{i=1}^{N_{1}}\left(\alpha_{i} \mathrm{Id}+\mathrm{D}\right)\right)\left(\prod_{k=1}^{N_{2}}\left(\mathrm{j} \omega_{k} \mathrm{Id}+\mathrm{D}\right)\right) .
$$

Let $\alpha \in \mathbb{C}$. When $\operatorname{Re}(\alpha) \neq 0$, the operator $(\alpha \mathrm{Id}+\mathrm{D})$ is easily invertible. If $\operatorname{Re}(\alpha)>0$, its inverse is the convolution operator with $\rho_{\alpha}(t):=\mathrm{e}^{-\alpha t} 1_{t \geq 0}$. We denote this inverse operator by $(\alpha \operatorname{Id}+\mathrm{D})^{-1}$. The case $\operatorname{Re}(\alpha)<0$ is treated in an identical manner. When $\alpha=\mathrm{j} \omega_{0}$ is purely imaginary, it is 
not possible to define an inverse that maps $\mathcal{S}(\mathbb{R})$ to itself. However, it is possible to define a left-inverse $J_{\omega_{0}}$ that is continuous from $\mathcal{S}(\mathbb{R})$ to $\mathcal{R}(\mathbb{R})$, the space of rapidly decaying measurable (not necessarily smooth) functions. The operator $\mathrm{J}_{\omega_{0}}$ is defined by

$$
\begin{aligned}
\mathrm{J}_{\omega_{0}} \varphi(t) & :=\mathrm{e}^{-\mathrm{j} \omega_{0} t} \int_{t}^{\infty} \mathrm{e}^{\mathrm{j} \omega_{0} \tau} \varphi(\tau) \mathrm{d} \tau \\
& -1_{t \geq 0} \mathrm{e}^{-\mathrm{j} \omega_{0} t} \int_{-\infty}^{\infty} \mathrm{e}^{\mathrm{j} \omega_{0} \tau} \varphi(\tau) \mathrm{d} \tau .
\end{aligned}
$$

Then, the operator

$$
P(\mathrm{D})^{*-1}:=\left(\prod_{k=1}^{N_{2}} \mathrm{~J}_{\omega_{k}}\right)\left(\prod_{i=1}^{N_{1}}\left(\alpha_{i} \mathrm{Id}+\mathrm{D}\right)^{-1}\right)
$$

is continuous from $\mathcal{S}(\mathbb{R})$ to $\mathcal{R}(\mathbb{R})$ and is a left-inverse of $P(\mathrm{D})^{*}$. Moreover, it has the following property, allowing for the definition of general CAR processes:

Proposition 3 (Theorem 4, [14]). Let $w$ be a white noise with Lévy-Schwartz exponent $f(\omega)$ and $P$ a polynomial. Then, the functional

$$
\exp \left(\int_{\mathbb{R}} f\left(P(\mathrm{D})^{*-1} \varphi(t)\right) \mathrm{d} t\right),
$$

where $P(D)^{*-1}$ is the operator defined below, is the characteristic functional of a generalized random process $s$.

The process $s$ satisfies the boundary conditions

$$
s(0)=\ldots=s^{\left(N_{1}\right)}(0)=0
$$

where $N_{1}$ is the number of purely imaginary roots of $P$, numbered with multiplicity.

Definition 4. The process $s$ defined in Proposition 3 is called an $\mathrm{AR}$ process and satisfies the equation $s=P(\mathrm{D})^{-1} w$.

Note that, with the notation of Proposition 3, a process $s=P(\mathrm{D})^{-1} w$ is stationary if $N_{1}=0$ and has stationary increments of order $N_{1}$ otherwise.

\section{E-SPlines ANd Generalized Poisson Processes}

\section{A. Non-Uniform Exponential Splines}

Exponential splines are the generalization of the polynomial B-splines introduced by Schoenberg [8]. B-splines are piecewise-polynomial functions. Similarly, exponential splines are functions that can be decomposed piecewise with exponential-polynomial monomials of the form $t^{m} \mathrm{e}^{\lambda t}$ with $m \in \mathbb{N}$ and $\lambda \in \mathbb{R}$. An E-spline is a continuous-domain function characterized by the discrete-domain vectors of its knots and coefficients. Moreover, the family of E-splines is rich enough to accurately approximate broad classes of functions. For these reasons, and due to their ease to manipulate, they are good candidates to connect the discrete and continuous worlds in signal processing [15].

Definition 5. A function $f$ is an exponential spline (or $E$ spline) if there exists a polynomial $P$ such that

$$
P(\mathrm{D}) f(t)=\sum_{k \in \mathbb{Z}} a_{k} \delta\left(t-t_{k}\right)
$$

for some $t_{k} \in \mathbb{R}$ (called the knots of $f$ ) and $a_{k} \in \mathbb{R}$. An exponential spline is said to be uniform if $t_{k}=k \tau$ for some $\tau>0$. Otherwise, it is said to be non-uniform.

Let $P=\prod_{n=1}^{N}\left(X+\alpha_{i}\right)$ with $\boldsymbol{\alpha}=\left(\alpha_{1}, \ldots, \alpha_{N}\right) \in \mathbb{R}^{N}$. As in Section II-C, we denote $\rho_{\alpha}(t):=\mathrm{e}^{-\alpha t} 1_{t \geq 0}$. Then, $\rho_{\boldsymbol{\alpha}}(t):=$ $\rho_{\alpha_{1}}(t) * \cdots * \rho_{\alpha_{N}}(t)$ is a Green function of $P(\mathrm{D})$. Then, if $p_{0}$ is in the null-space of $P(D)$,

$$
f(t)=\sum_{k \in \mathbb{Z}} a_{k} \rho_{\boldsymbol{\alpha}}\left(t-t_{k}\right)+p_{0}(t)
$$

is an E-spline associated with $P$. Conversely, every E-spline has the latter form.

\section{B. Poisson driven CAR Processes as Random E-Splines}

In general, sample paths of CAR processes do not have a finite number of degrees of freedom per unit of time. Following the terminology of [7], we say that they have an infinite rate of innovation. However, there is a subfamily of processes that have a finite rate of innovation: those driven by compound Poisson noises. The realizations of these processes are exponential splines.

An infinitely divisible random variable $X$ is a compound Poisson random variable if its Lévy exponent has the form

$$
f(\omega)=\lambda(\widehat{p}(\omega)-1)
$$

with $\lambda>0$ and $\widehat{p}$ the characteristic function of a probability measure $p$ on $\mathbb{R} \backslash\{0\}$. Equivalently, the probability law of $X$ is given by

$$
\mathrm{e}^{-\lambda}\left(\delta+\sum_{n \in \mathbb{N} \backslash\{0\}} \lambda^{n} p^{* n} / n !\right),
$$

with $\delta$ the Dirac impulse, $p^{* 1}:=p$, and $p^{*(n+1)}:=p^{* n} * p$.

Definition 6. An impulsive noise (or compound Poisson noise) is a Lévy white noise whose Lévy exponent has the form (13).

$A$ generalized Poisson process $s$ is an CAR process $s=$ $P(\mathrm{D})^{-1} w$ driven by an impulsive noise $w$.

A process driven by an impulsive noise is called a generalized Poisson process, for it generalizes the family of compound Poisson processes (when $P=X$ i.e., $\mathrm{D} s=w$ ). The previous definition in terms of Lévy exponents is quite abstract but can be made explicit. Indeed, if $w$ is an impulsive noise, one has [6]

$$
w(t)=\sum_{n \in \mathbb{N}} a_{n} \delta\left(t-t_{n}\right),
$$

where the $a_{n}$ are i.i.d. with common law $\mathbb{P}$ and, for all $a<b$, the $t_{n}$ are such that $\operatorname{Prob}\left(\sharp\left\{n, a \leq t_{n} \leq b\right\}=\right.$ $k)=\mathrm{e}^{-\lambda(b-a)} \lambda^{k}(b-a)^{k} / k$ ! (Poisson random variable of parameter $\lambda(b-a))$. This property of being a stream of Dirac impulses explains the terminology "impulsive" behind compound Poisson noises. A direct consequence of this result is given by the following: 
Proposition 7. A generalized Poisson process $s=P(\mathrm{D})^{-1} w$ is a random non-uniform exponential spline. In other words, there exists a polynomial $P$ such that

$$
P(\mathrm{D}) s(t)=\sum_{n \in \mathbb{Z}} a_{n} \delta\left(t-t_{n}\right),
$$

where the $a_{n}$ and the $t_{n}$ are like in (15). This implies that we have

$$
s(t)=\sum_{n \in \mathbb{Z}} a_{n} \rho_{\boldsymbol{\alpha}}\left(t-t_{n}\right)+p_{0}(t),
$$

where $\rho_{\boldsymbol{\alpha}}(t)$ is a Green function of $P(\mathrm{D})$ and $p_{0}(t)$ is a random element of the finite-dimensional null space of $P(D)$.

\section{CAR PROCESSES AS LIMITS OF RANDOM E-SPLINES}

In this section, we expose our main result, which can be seen as a generalization into an infinite-dimensional setting of the following well-known fact: every infinitely divisible random variable is the limit in distribution of a sequence of compound Poisson random variables [12, Corollary 8.8].

Recall that a sequence of random vectors in $\mathbb{R}^{N}$, denoted by $\left(\boldsymbol{X}_{n}\right)_{n \in \mathbb{N}}$, converges in distribution to $\boldsymbol{X}$ if $\operatorname{Prob}\left(\boldsymbol{X}_{n} \in\right.$ $B) \underset{n \rightarrow \infty}{\longrightarrow} \operatorname{Prob}(\boldsymbol{X} \in B)$ for every Borel set $B \in \mathbb{R}^{N}$. Similarly, we say that the sequence of generalized random processes $s_{n}$ converges in distribution to $s$ if for every $N \in \mathbb{N}$ and $\left(\varphi_{1}, \ldots, \varphi_{N}\right) \in \mathcal{S}(\mathbb{R})^{N}$, the sequence of random vectors $\boldsymbol{X}_{n}=\left(\left\langle s_{n}, \varphi_{1}\right\rangle, \ldots,\left\langle s_{n}, \varphi_{N}\right\rangle\right)$ converges in distribution to $\boldsymbol{X}=\left(\left\langle s, \varphi_{1}\right\rangle, \ldots,\left\langle s, \varphi_{N}\right\rangle\right)$. The idea behind this concept is that all the statistics (finite-dimensional marginals, moments, etc.) of the limit process $s$ are the limits of the statistics of the $s_{n}$.

Theorem 8. Let $s=P(\mathrm{D})^{-1} w$ be a CAR process. There exists a sequence of generalized Poisson processes $s_{n}=P(\mathrm{D})^{-1} w_{n}$ such that

$$
s_{n} \underset{n \rightarrow \infty}{\longrightarrow} s
$$

in distribution.

Combining Proposition 7 and Theorem 8, we deduce that a CAR process is the limit in law of random non-uniform exponential splines. Note that those random exponential splines are very particular: their knots $t_{n}$ are distributed according to a Poisson measure and the weights $a_{n}$ are i.i.d.

The sketch of the proof of Theorem 8 is a follows:

- Let $f(\omega)$ be a Lévy-Schwartz exponent and $\left(\lambda_{n}\right)$ a sequence such that $\lambda_{n} \underset{n \rightarrow \infty}{\longrightarrow}+\infty$. Let $f_{n}(\omega):=$ $\lambda_{n}\left(\mathrm{e}^{f(\omega) / \lambda_{n}}-1\right)$. According to Proposition 1, $\mathrm{e}^{f(\omega) / \lambda_{n}}$ is the characteristic function of a probability law $\mathbb{P}_{n}$. Hence, $f_{n}(\omega)=\lambda_{n}\left(\widehat{\mathbb{P}}_{n}(\omega)-1\right)$ is the Lévy exponent of an impulsive noise $w_{n}$ according to (13).

- From the relation $f_{n}(\omega) \underset{n \rightarrow \infty}{\longrightarrow} f(\omega)$, one can deduce, using the techniques of [13], that $\widehat{\mathscr{P}}_{w_{n}}(\psi) \underset{n \rightarrow \infty}{\longrightarrow} \widehat{\mathscr{P}}_{w}(\psi)$ for every $\psi \in \mathcal{R}(\mathbb{R})$, the space of rapidly decaying function (see Section II-C).

- We base our result on the following infinite dimensional generalization the Lévy continuity theorem:
Theorem 9 ([16]). A sequence of generalized random processes $s_{n}$ in $\mathcal{S}^{\prime}(\mathbb{R})$ converges in distribution to a generalized random process $s$ if and only if

$$
\widehat{\mathscr{P}}_{s_{n}}(\varphi) \underset{n \rightarrow \infty}{\longrightarrow} \widehat{\mathscr{P}}_{s}(\varphi)
$$

for every $\varphi \in \mathcal{S}(\mathbb{R})$.

The nuclear structure of $\mathcal{S}(\mathbb{R})$ is the reason why this result holds.

- Let $\varphi \in \mathcal{S}(\mathbb{R})$. Since $\psi:=P(\mathrm{D})^{-1 *} \varphi \in \mathcal{R}(\mathbb{R})$, one has

$$
\begin{aligned}
& \widehat{\mathscr{P}}_{s_{n}}(\varphi)=\widehat{\mathscr{P}}_{w_{n}}\left(P(\mathrm{D})^{-1 *} \varphi\right)=\widehat{\mathscr{P}}_{w_{n}}(\psi) \\
& \underset{n \rightarrow \infty}{\longrightarrow} \widehat{\mathscr{P}}_{w}(\psi)=\widehat{\mathscr{P}}_{s}(\varphi) .
\end{aligned}
$$

which is equivalent to Theorem 8 according to Theorem 9.

Note that the previously exposed convergence scheme holds as $\lambda_{n} \rightarrow+\infty$. For a generalized Poisson process, the parameter $\lambda$ measures the average number of knots per unit of time. Hence, a CAR process $s$ is approximated by random E-splines with increasing numbers of jumps (in average) per unit of time $\lambda_{n}$. This is coherent with the fact that a non-Poisson CAR process has an infinite rate of innovation.

\section{Simulations}

We represent realizations of CAR processes and of their statistical approximation by generalized Poisson processes in Figure 1. The approximation scheme is the same as the one exposed in the sketch of the proof of Theorem 8. We consider different CAR processes whitened by the operators $(\mathrm{D}-\alpha \mathrm{Id}),(\mathrm{D}-\alpha \mathrm{Id})^{2}$, or $\mathrm{D}$. The latter operator defines a Lévy process, whereas the two others correspond to stationary CAR processes. We also consider different Lévy white noises: the Gaussian white noise, with Lévy exponent $f(\omega)=-\omega^{2} / 2$, and the Cauchy white noise, which is a member of the family of $\mathrm{S} \alpha \mathrm{S}$ processes [9], [17] with Lévy exponent $f(\omega)=-|\omega|$. For a given process $s=P(\mathrm{D})^{-1} w$ with Lévy exponent $f(\omega)$, we generate generalized Poisson processes with Lévy exponent $f_{n}(\omega)=\lambda_{n}\left(\mathrm{e}^{f(\omega) / \lambda_{n}}\right)$ for increasing values of $\lambda_{n}$. Note that the probability law of the jumps of the Poisson processes that approximate Gaussian (Cauchy, respectively) processes follow a Gaussian (Cauchy, respectively) distribution.

As we see in Figure 1, for small values of $\lambda_{n}$, we recognize that the realizations of generalized Poisson processes are exponential splines. For large values of $\lambda_{n}$-of order $10^{4}$ in our simulations - the realization of a generalized Poisson process is statistically indistinguishable from the realization of the corresponding CAR process. These two facts are coherent with our theoretical results.

\section{CONCLUSION}

We have shown that any continuous-domain CAR process (including Gaussian and sparse type processes) is the limit in distribution of generalized Poisson processes. Since the realizations of generalized Poisson processes are random nonuniform E-splines, we provided a new interpretation of CAR processes. This gives in particular a new way to synthesize CAR processes as random E-splines by approximation. 

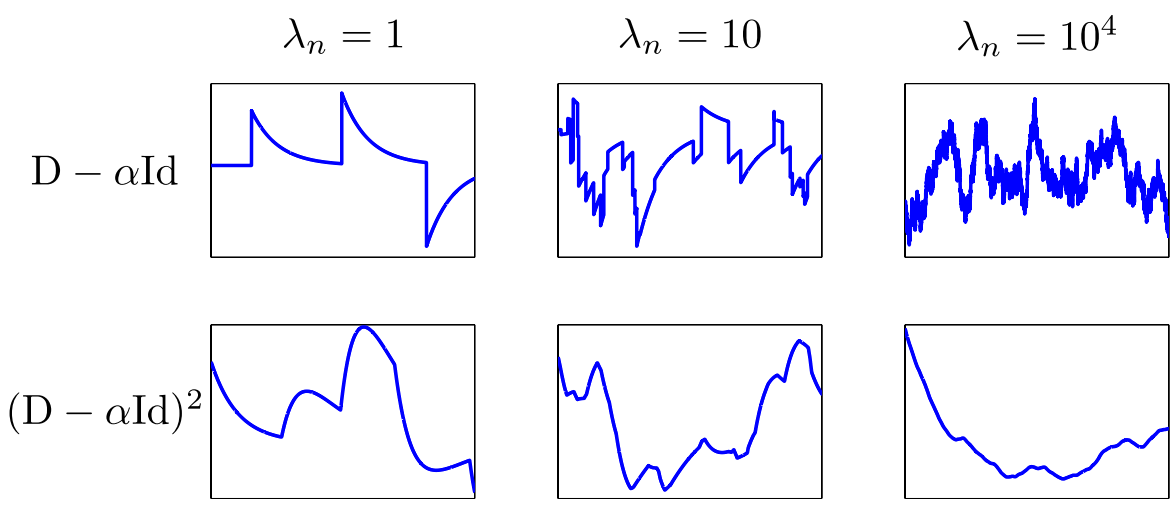

$\lambda_{n}=10^{4}$
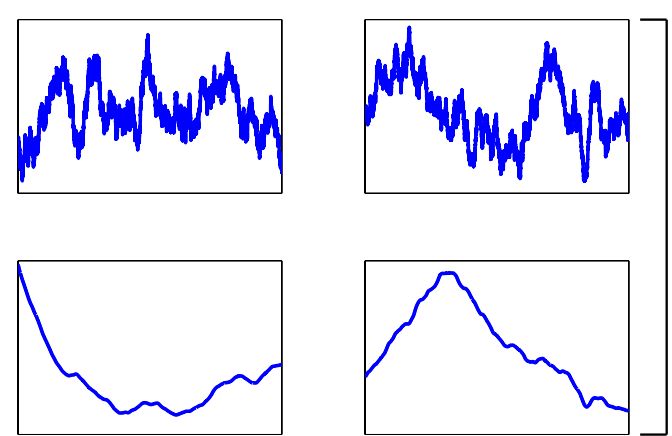

Gaussian
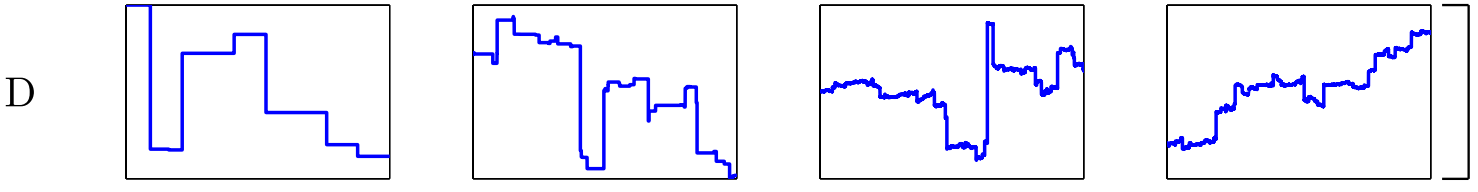

Cauchy

Fig. 1. Convergence in distribution of generalized Poisson processes to CAR processes. In the last column, the processes to be approximated: from top to bottom, $s=(\mathrm{D}-\alpha \mathrm{Id})^{-1} w_{\text {Gaussian }} s=(\mathrm{D}-\alpha \mathrm{Id})^{-2} w_{\text {Gaussian }}$ where $\alpha>0$, and $s=\mathrm{D}^{-1} w_{\mathrm{Cauchy}}$. In the first three columns, the associated generalized Poisson processes with increasing $\lambda_{n}$.

\section{ACKNOWLEDGMENT}

The research leading to these results has received funding from the European Research Council under the European Union's Seventh Framework Programme (FP7/2007-2013) / ERC grant agreement $n^{\circ} 267439$.

\section{REFERENCES}

[1] R. Gray and L. Davisson, An introduction to statistical signal processing. Cambridge University Press Cambridge, 2004, vol. 49.

[2] M. Unser, P. Tafti, A. Amini, and H. Kirshner, "A unified formulation of Gaussian versus sparse stochastic processes-Part II: Discrete-domain theory," Information Theory IEEE Transactions, vol. 60, no. 5, pp. 30363051, May 2014.

[3] H. Kirshner, M. Unser, and J. Ward, "On the unique identification of continuous-time autoregressive models from sampled data," IEEE Transactions on Signal Processing, vol. 62, no. 6, pp. 1361-1376, March 15, 2014.

[4] P. Pad and M. Unser, "Optimality of operator-like wavelets for representing sparse AR(1) processes," CoRR, vol. abs/1312.1147, 2013. [Online]. Available: http://arxiv.org/abs/1312.1147

[5] B. Mandelbrot, "The fractal geometry of nature. 1982," San Francisco, CA, 1982.

[6] M. Unser and P. Tafti, "Stochastic models for sparse and piecewisesmooth signals," Signal Processing IEEE Transactions, vol. 59, no. 3, pp. 989-1006, March 2011.

[7] M. Vetterli, P. Marziliano, and T. Blu, "Sampling signals with finite rate of innovation," Signal Processing, IEEE Transactions on, vol. 50, no. 6, pp. 1417-1428, 2002.

[8] M. Unser and T. Blu, "Cardinal exponential splines: Part I-Theory and filtering algorithms," IEEE Transactions on Signal Processing, vol. 53, no. 4, pp. 1425-1438, April 2005.

[9] M. Unser and P. Tafti, An Introduction to Sparse Stochastic Processes. Cambridge, UK: Cambridge University Press, 2014, 367 p.

[10] H. W. Bode and C. E. Shannon, "A simplified derivation of linear least square smoothing and prediction theory," Proceedings of the IRE, vol. 38, no. 4, pp. 417-425, 1950.

[11] I. Gel'fand and N. Vilenkin, Generalized functions. Vol. 4: Applications of harmonic analysis, ser. Translated by Amiel Feinstein. Academic Press, New York - London, 1964, 1964.
[12] K.-I. Sato, Lévy processes and infinitely divisible distributions, ser. Cambridge Studies in Advanced Mathematics. Cambridge University Press, Cambridge, 2013, vol. 68, translated from the 1990 Japanese original, Revised edition of the 1999 English translation.

[13] J. Fageot, A. Amini, and M. Unser, "On the continuity of characteristic functionals and sparse stochastic modeling," Journal of Fourier Analysis and Applications, vol. 20, pp. 1179-1211, 2014.

[14] M. Unser, P. Tafti, and Q. Sun, "A unified formulation of Gaussian versus sparse stochastic processes-Part I: Continuous-domain theory," Information Theory IEEE Transactions, vol. 60, no. 3, pp. 1945-1962, March 2014.

[15] M. Unser, "Cardinal exponential splines: Part II-Think analog, act digital," IEEE Transactions on Signal Processing, vol. 53, no. 4, pp. 1439-1449, April 2005.

[16] P. Boulicaut, "Convergence cylindrique et convergence étroite d'une suite de probabilités de Radon," Z. Wahrscheinlichkeitstheorie und Verw. Gebiete, vol. 28, pp. 43-52, 1973/74.

[17] G. Samorodnitsky and M. Taqqu, Stable non-Gaussian processes: Stochastic models with infinite variance. Chapman and Hall, 1994. 